\title{
The role of gut-associated lymphoid tissues and mucosal defence
}

\author{
Maria Luisa Forchielli ${ }^{1,2}$ and W. Allan Walker ${ }^{2,3 *}$ \\ ${ }^{1}$ Department of Pediatrics, University of Bologna, Bologna, Italy \\ ${ }^{2}$ Department of Pediatrics, Harvard Medical School, Boston, MA, USA \\ ${ }^{3}$ Harvard Medical School, Mucosal Immunology Laboratories, Massachusetts General Hospital, Boston, MA, USA
}

\begin{abstract}
The newborn infant leaves a germ-free intrauterine environment to enter a contaminated extrauterine world and must have adequate intestinal defences to prevent the expression of clinical gastrointestinal disease states. Although the intestinal mucosal immune system is fully developed after a full-term birth, the actual protective function of the gut requires the microbial stimulation of initial bacterial colonization. Breast milk contains prebiotic oligosaccharides, like inulin-type fructans, which are not digested in the small intestine but enter the colon as intact large carbohydrates that are then fermented by the resident bacteria to produce SCFA. The nature of this fermentation and the consequent $\mathrm{pH}$ of the intestinal contents dictate proliferation of specific resident bacteria. For example, breast milk-fed infants with prebiotics present in breast milk produce an increased proliferation of bifidobacteria and lactobacilli (probiotics), whereas formula-fed infants produce more enterococci and enterobacteria. Probiotics, stimulated by prebiotic fermentation, are important to the development and sustainment of intestinal defences. For example, probiotics can stimulate the synthesis and secretion of polymeric IgA, the antibody that coats and protects mucosal surfaces against harmful bacterial invasion. In addition, appropriate colonization with probiotics helps to produce a balanced $\mathrm{T}$ helper cell response $(\mathrm{Th} 1=\mathrm{Th} 2=\mathrm{Th} 3 / \mathrm{Tr} 1)$ and prevent an imbalance $(\mathrm{Th} 1>\mathrm{Th} 2$ or $\mathrm{Th} 2>\mathrm{Th} 1)$ contributing in part to clinical disease (Th2 imbalance contributes to atopic disease and Th1 imbalance contributes to Crohn's disease and Helicobacter pylori-induced gastritis). Furthermore, a series of pattern recognition receptors, toll-like receptors on gut lymphoid and epithelial cells that interact with bacterial molecular patterns (e.g. endotoxin (lipopolysaccharide), flagellin, etc.), help modulate intestinal innate immunity and an appropriate adaptive immune response. Animal and clinical studies have shown that inulin-type fructans will stimulate an increase in probiotics (commensal bacteria) and these bacteria have been shown to modulate the development and persistence of appropriate mucosal immune responses. However, additional studies are needed to show that prebiotics can directly or indirectly stimulate intestinal host defences. If this can be demonstrated, then prebiotics can be used as a dietary supplement to stimulate a balanced and an appropriately effective mucosal immune system in newborns and infants.
\end{abstract}

Development of gut immunity: Prebiotics and intestinal host defence: Prebiotics and microbial function

The notion that prebiotics may help in the development of intestinal defences developed as a result of the observation that newborns, including full-term newborns, lack an adequate capacity to defend themselves against a variety of intestinal infections because of an incompletely developed intestinal host defence system. Although the efferent components (Peyer's patches (PP), $\mathrm{M}$ cells, mucosal and intraepithelial lymphocytes) of the mucosal immune system are complete after a full gestation, the gut requires initial bacterial colonization, which occurs in the extrauterine environment, in order to respond to a subsequent pathogen/infectious stimulus. Breast milk contains multiple protective nutrients and active factors, including non-digestible oligosaccharides, and has been shown to passively and actively protect the newborn from harmful, age-related intestinal infections (Clavano, 1982).

Since a major portion of the human milk carbohydrates consist of a variety of non-digestible oligosaccharides that enter the colon to be fermented and create a luminal environment (acid $\mathrm{pH}$ and increased SCFA) that favours the proliferation of probiotic/ commensal bacteria, lactobacillus and bifidobacteria, it is assumed that these factors in breast milk (prebiotics) favour the development of an appropriate colonization process that in turn stimulates the maturation of intestinal host defences (Yoshioka et al. 1983). This review first considers the importance of appropriate bacterial colonization in the development of host defence, the role of probiotics in this process, and then reviews the published studies which suggest that prebiotics contribute to the host defences. We close with suggested additional studies that are needed to confirm the effects of prebiotics on gut-associated lymphoid tissues (GALT) and intestinal host defence.

\section{Gut-associated lymphoid tissues}

The GALT is the largest collection of lymphoid tissues in the body, consisting of both organized lymphoid tissues, such as mesenteric lymph nodes (MLN) and PP, and more diffusely scattered lymphocytes in the intestinal lamina propria (LP) and epithelium including

Abbreviations: APC, antigen-presenting cells; DC, dendritic cells; DSS, dextran sulphate sodium; FAE, follicle-associated epithelium; GALT, gut-associated lymphoid tissues; IBD, inflammatory bowel disease; IEC, intestinal epithelial cells; INF- $\gamma$, interferon- $\gamma$; LP, lamina propria; LPS, lipopolysaccharide; MLN, mesenteric lymph nodes; NEC, necrotizing enterocolitis; PAMP, pathogen-associated molecular pattern; PBMC, peripheral blood mononuclear cells; PGN, peptidoglycan; PP, Peyer's pathches; TGF- $\beta$, transforming growth factor- $\beta$; Th, T helper; TLR, toll-like receptor; Tollip, toll inhibitory protein; Treg, T regulatory cells. 
large numbers of $\operatorname{IgA}^{+}$plasmablasts. Polymeric $\operatorname{IgA}$, one of the hallmarks of the intestinal humoral immune system, is produced to defend mucosal surfaces from environmental microbes. Germ-free mice have a reduced number of IgA-secreting-plasmocytes (Moreau et al. 1988). Monocolonization with segmented filamentous bacteria, a major component of commensal Grampositive bacteria, induces a robust mucosal $\operatorname{IgA}$ response in PP and LP (Talham et al. 1999). It was suggested that specific intestinal mucosal IgA can be induced by commensal bacterial antigens through a $\mathrm{T}$ cell-independent pathway and is independent of influence by follicular organized lymphoid tissue (MacPherson et al. 2001).

It is well established that colonization with bacteria is critical for the normal structural and functional development and optimal function of the mucosal immune system. As demonstrated by early studies, germ-free mice exhibit smaller PP (Moreau \& Corthier, 1988) and fewer intraepithelial lymphocytes (Lefrancois \& Goodman, 1989), compared to specific pathogen-free or germfree mice colonized with single or multiple species of bacteria. A recent study using gnotobiotic rats showed that under germfree conditions, the follicle-associated epithelium (FAE) contains only a few T and B cells and the B cells were negative for CD86, a co-stimulatory molecule necessary for $\mathrm{T}$ cell activation. After bacterial colonization, however, the number of $\mathrm{T}$ cells and $\mathrm{CD} 6^{+} \mathrm{B}$ cells in the FAE increase dramatically (Yamanaka et al. 2003). Furthermore, this study showed that before microbial colonization, immature $\left(\mathrm{CD} 80^{-}\right.$or $\left.\mathrm{CD}^{-} 6^{-}\right) \mathrm{CD}^{+}$dendritic cell (DC) subsets were detected in the FAE, and this subset disappeared from the FAE soon after colonization, probably as a result of rapid DC migration to the inter-follicular $\mathrm{T}$ cell zone where DC activation/maturation and stimulation of naive $\mathrm{T}$ cells are known to take place. The results of this study, therefore, suggest the involvement of microbial colonization in organizing the spatial relationships of $\mathrm{B}$ cells, $\mathrm{T}$ cells and $\mathrm{DC}$ in PP (Yamanaka et al. 2003). Luminal bacteria also affect intestinal antigen-transporting $\mathrm{M}$ cells. Colonization of germ-free mice with Salmonella typhymurium aroA results in a 2- to 3-fold increase in the number of M cells (Savidge et al. 1991).

One of the key functions of GALT is to distinguish innocuous antigens from pathogenic micro-organisms and to elicit an appropriate response. These responses can be affected significantly by the intestinal microflora. Intestinal bacterial antigens are believed to play a role in the induction of $\mathrm{T}$ cell activation as the majority of $\mathrm{T}$ lymphocytes from the intestinal lymphoid tissues show an activated or memory phenotype. Monocolonization of germ-free animals can result in the activation of mucosal $\mathrm{T}$ cells that, under germ-free conditions, are quiescent. Unrestrained mucosal immune activation in response to bacterial signals from the lumen is, however, a risk factor for inflammatory diseases, such as inflammatory bowel disease (IBD). The development and function of GALT are precisely regulated by mechanisms that are still not fully understood. It has been thought that regulatory $\mathrm{T}$ cells may play a role in the modulation of intestinal mucosal immune responses. In the absence of $\mathrm{T}$ regulatory cells (Treg), gut bacteria act as a trigger for the development of severe IBD. As shown in an animal study, transfer of $\mathrm{CD}^{+} \mathrm{T}$ cells to immune-deficient mice in the absence of a $\mathrm{CD} 25^{+}$subset leads to colitis, if enteric bacteria colonize the hosts. However, adoptive transfer of regulatory $\mathrm{T}$ cells into these hosts prevents the expression of disease (Mottet et al. 2003). Failure to precisely regulate the mucosal immune system may also result in the development of an immune response to food proteins (allergy) or the induction of a destructive response directly against selfantigens (autoimmune disease). Although intestinal $\mathrm{T}$ cells express an activated phenotype and are enriched for memory cells, they proliferate poorly in vitro in response to $\mathrm{T}$ cell stimulation. Human mucosal $\mathrm{CD} 4^{+} \mathrm{T}$ cells have been shown to suppress proliferation of peripheral blood $\mathrm{T}$ cells in response to antigen pre-pulsed antigen-presenting cells (APC) in a co-culture system (Khoo et al. 1997). This inhibition was independent of cell contact, and was reversible by neutralization of IL-10 or transforming growth factor- $\beta$ (TGF- $\beta$ ) with monoclonal antibody, suggesting that specific antigen recognition may play a role in maintaining mucosal $\mathrm{T}$ cell unresponsiveness to intestinal bacterial antigens. Germ-free animals have been shown to be partially or totally resistant to the induction of oral tolerance to some antigens (Moreau \& Corthier, 1988; Wannemuehler et al. 1992; Moreau \& Gaboriau-Routhiau, 1996; Sudo et al. 1997). Priming of helper T cells by bacterial antigens in GALT has also been suggested to play a role in determining the ability of these cells to rapidly respond to the cognate antigen in the peripheral lymphoid organs (Julia et al. 2000).

GALT can be divided into discrete inductive and effector sites, consisting of PP, LP and MLN and contains both $\mathrm{CD}^{+}$and $\mathrm{CD}^{+} \mathrm{T}$ cells. A subpopulation of $\mathrm{CD} 4^{+} \mathrm{T}$ cells that co-express CD25 (the $\alpha$-chain of IL-2R) with regulatory function has been identified in the mucosa, and has been purported to be generated by the exposure of intestinal immune system to luminal antigens. These cells appear to play an important role in the induction and maintenance of tolerance in the normal intestine through the production of TGF- $\beta$ and IL-10 and the ligation of cytotoxic T lymphocyte-associated antigen-4 (Shevach, 2000). As discussed earlier, Treg $\left(\mathrm{CD}^{+}{ }^{+} \mathrm{CD} 45 \mathrm{RB}^{\mathrm{lo}}, \mathrm{CD}^{+} \mathrm{CD} 25^{+}\right)$are potent immune suppressors that control or prevent the development of spontaneous autoimmune diseases (Suri-Payer et al. 1998) and are involved in preventing intestinal IBD (Read et al. 2000), in which gut mucosal $\mathrm{T}$ cells develop an excessive immune response to intestinal microflora. A recent study examined the role of microbial products in activating these regulatory cells and showed that Treg respond directly to pro-inflammatory bacterial products (lipopolysaccharide (LPS)). LPS treatment enhances the suppressive function of these cells via the TLR4 receptor molecule (Caramalho et al. 2003). In addition, this study also demonstrated that a subset of $\mathrm{CD} 4\left(\mathrm{CD} 45 \mathrm{RB}^{\mathrm{lo}} \mathrm{CD} 25^{+}\right)$cells selectively express toll-like receptors (TLR) 4, 5, 7, and 8 (24), providing a link between the innate and adaptive immune responses. Fig. 1 depicts the various components of the mucosal immune system in the gut necessary to provide protection.

\section{Bacterial-epithelial 'crosstalk' and bacterial antigen uptake}

Colonization with normal bacterial flora in the gastrointestinal tract is often cited as a basic defence mechanism of the body against infections by pathogens (Cebra, 1999; Falk et al. 1999). For this process to occur, the colonizing bacteria must communicate with the underlying epithelium. This communication leads to metabolic/immunologic reactions by the epithelial cell and its underlying lymphoid cells. The communication between intestinal bacteria and the gut is termed microbial-epithelial 'crosstalk'. (Fig. 2). Animal studies show that gnotobiotic mice or rats were much more susceptible than their conventionally reared counterparts to infection by Salmonella enteritidis, 


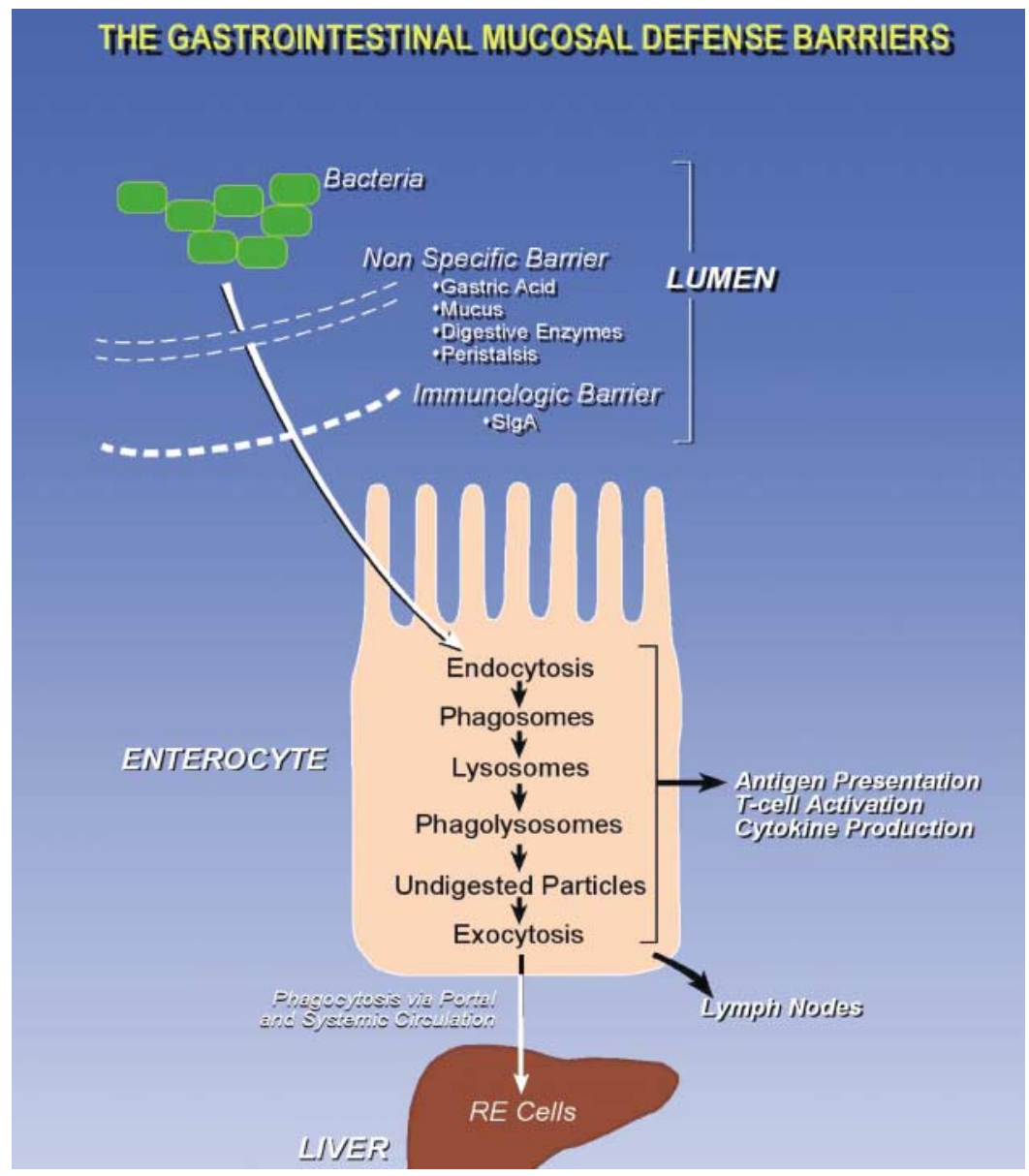

Fig. 1. Cartoon of non-immunologic and immunologic components of gastrointestinal defence against noxious luminal stimuli. These components work in concert to protect the intestinal surface from continuous interaction with foreign antigens and micro-organisms. Function requires adequate colonization.

Listeria monocytogenes, Clostridium difficile and Helicobacter pylori. Neonatal animals and infants are more susceptible to infections than adults, which may be related to the change in the composition and number of gut flora after weaning, effecting a greater resistance to infection seen in adults. Alternatively, changes in the mucosal immune system that occur at weaning, due to the changes in gut flora, may also be responsible (Steege et al. 1997; Cebra, 1999). Another component of initial colonization is the stimulation of T helper (Th) cells to produce a balanced Th response $(\mathrm{Th} 1=\mathrm{Th} 2=\mathrm{Th} 3$; Table 1$)$.

Colonization of intestinal flora begins at birth. The maternal microflora can be a source of bacteria colonizing the newborn's intestine. Colonization can also be affected by environmental factors and by infant feeding patterns. Breast-feeding is found to encourage the growth of bifidobacteria, whereas formula-fed infants have a more complex flora made up of bifidobacteria, bacteroides, clostridia and streptococci. Bacteria may use microvillus membrane glycoconjugates as target cell receptors for adherence to the gastrointestinal surface. In mice intestine, activities of glycosyltransferases, the enzymes that are responsible for synthesizing brush border glycoconjugates, are under tissue-specific and developmental regulation (Nanthakumar et al. 2003). The intestinal flora has been shown to participate in regulating specific glycosyltransferases (Nanthakumar et al. 2003). Even though intestinal epithelial cells are normally densely colonized by

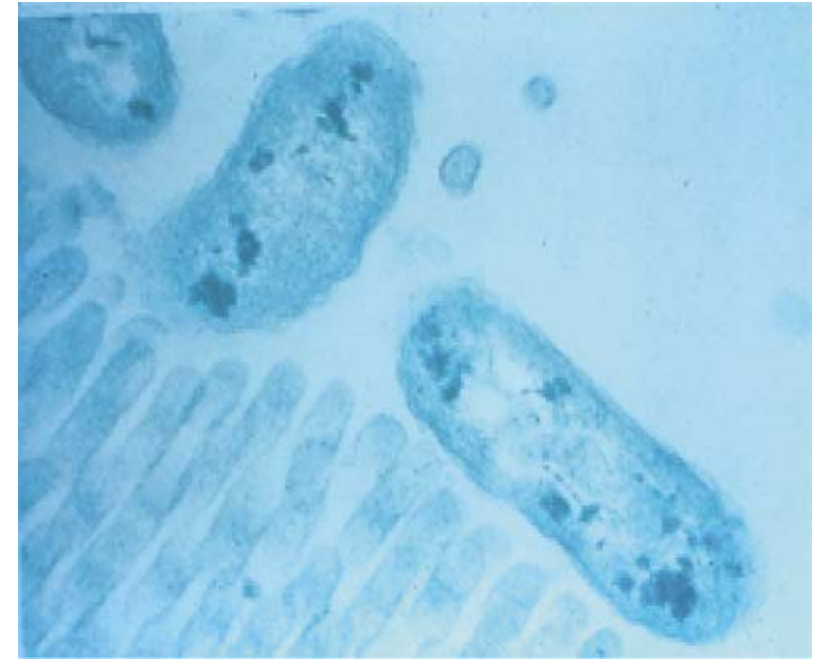

Fig. 2. An electron microscopic view of an enteric bacterium interacting with the microvillus surface of the small intestine, e.g. microbial-epithelial 'crosstalk'. This communication represents an important stimulus to the development of host defence. 
Table 1. Role of bacterial colonization in a balanced T helper cell response

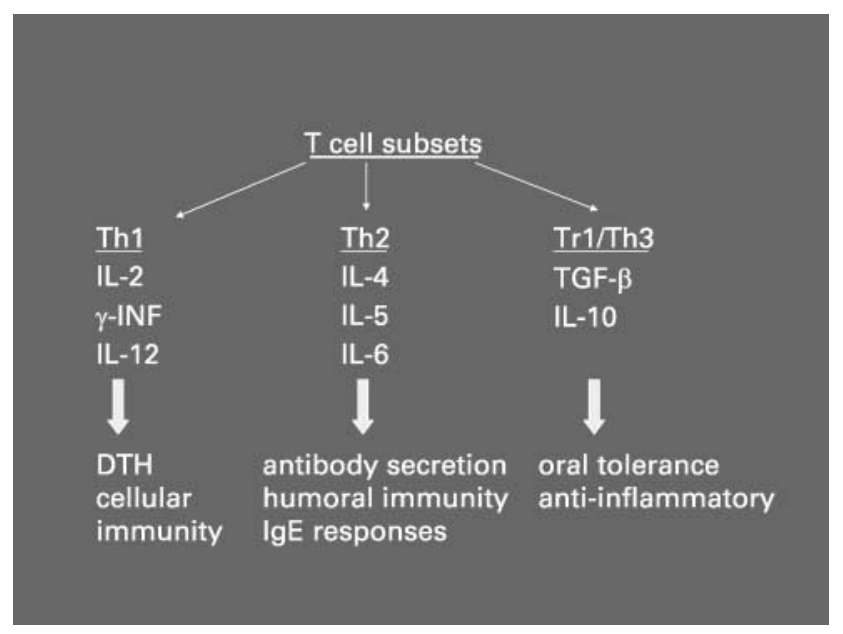

a wide variety of microbes (up to 500 species of bacteria), they form a mechanical barrier that separates the external environment from the host's internal milieu. Intestinal epithelial cells (IEC) are also capable of detecting bacterial antigens (or products) and initiating, participating in or regulating both innate as well as adaptive immune responses by transducing signals from luminal pathogens to adjacent immune cells present in the LP, such as the macrophages, DC and lymphocytes that form the intestinal mucosal immune system, via molecules expressed on the epithelial cell surface, such as MHC I, MHC II and TLR (Cario \& Podolsky, 2000; Hershberg \& Mayer, 2000; Fusunyan et al. 2001; Cario et al. 2002). TLR on the epithelial cells may enable the epithelium to discriminate between normal commensal flora and pathogens and induce the transcription activation of a panel of genes mediating immune and inflammatory responses. TLR on IEC therefore play an important role in the intestinal epithelial innate immune signalling pathways and in initiating and regulating adaptive immune responses.

TLR are a family of transmembrane receptors homologous to Drosophila toll proteins (Medzhitov et al. 1997; Akira et al. 2001; Mushegian \& Medzhitov, 2001). Recognition of bacterial molecular patterns by the innate immune system is dependent on interaction between TLR and pathogen-associated molecular patterns (PAMP) present on diverse microbes. To date, ten members of the TLR family have been identified in mammals, with each receptor recognizing a unique set of PAMP (Table 2). TLR2 is responsible for the recognition of bacterial lipoproteins and peptidoglycan (PGN), TLR3 recognizes double-stranded RNA TLR4

Table 2. Toll-like receptors (TLR) and their ligands

\begin{tabular}{ll}
\hline TLR & Ligands \\
\hline TLR1 & Triacyl lipopeptides \\
TLR2 & Lipoprotein/lipopeptides, peptidoglycan, lipoteichoic acid, etc. \\
TLR3 & Double-stranded RNA \\
TLR4 & Lipopolysaccharide, HSP60 etc., commensal bacteria \\
TLR5 & Flagellin \\
TLR6 & Diacyl lipopeptides \\
TLR7 & Synthetic compounds (the immune response modifiers) \\
TLR8 & Unknown \\
TLR9 & CpG DNA \\
TLR10 & Unknown \\
\hline
\end{tabular}

is essential for responses to LPS, TLR5 controls responses to bacterial flagellin, TLR7 responds to the immune response modifiers (imidazoquinolines), and TLR9 is required for the recognition of unmethylated CpG DNA motifs characteristic of bacterial DNA. Recent studies have suggested that TLR may control the induction of adaptive Th1 responses and there is also evidence that TLR may be important for Th2-type responses by augmenting the overall maturity of DC (Moreau \& Corthier, 1988; Dabbag \& Lewis, 2003; Table 1).

Intestinal epithelium, which is continually exposed to a large variety of commensal bacteria and PAMP, has been shown to constitutively express several members of the TLR in vitro and in vivo (Cario \& Podolsky, 2000; Gewirtz et al. 2001; Cario et al. 2002; Melmed et al. 2003; Nanthakumar et al. 2003). It is well established that the interaction of TLR with its PAMP results in the activation of intracellular signalling pathways, nuclear translocation of transcription factor, NF- $\mathrm{\kappa B}$ and the transcription of cytokines such as IL-8. Cario \& Podolsky (2000) characterized the expression pattern of TLR2, TLR3, TLR4 and TLR5 in the intestinal mucosa of IBD patients compared with normal controls. They observed a differential expression of TLR in the IEC using immunohistochemistry, showing a constitutive expression of TLR2 and TLR5, and a low level expression of TLR4 in normal cells. In contrast, IEC of IBD patients showed an up-regulated expression of TLR4, down-regulated expression of TLR3 and unchanged TLR2 and TLR5 expression (Cario \& Podolsky, 2000). A study by Fusunyan et al. (2001) showed that on a non-malignant human fetal primary small intestinal cell line (H4 cells) and human fetal small intestinal enterocytes TLR2 and TLR4 were expressed constitutively, and that the transcription of TLR 2 and TLR4 on immature intestinal enterocytes was regulated by inflammatory stimuli. The authors observed that IL-1 $\beta$ stimulation resulted in an increased expression of both TLR 2 and TLR4, whereas LPS decreased TLR4 expression on $\mathrm{H} 4$ cells and on human fetal small intestinal enterocytes. However, a study by the Sanderson group showed that TLR4 mRNA was expressed in peripheral blood mononuclear cells (PBMC), but not present in the epithelium from children with IBD (Naik et al. 2001).

The expression of TLR in IEC has also been studied in an animal model. Ortega-Cava et al. (2003) examined the localization of TLR4 and compared it with that of TLR2 and CD14 along the luminal gastrointestinal surface of normal mice and mice with dextran sulphate sodium (DSS)-induced IBD. They found that TLR are differentially expressed along the mouse gastrointestinal tract with higher levels of TLR4 and CD14 mRNA and protein detected in the distal colon and a stronger TLR 2 mRNA expression found in the proximal colon. The authors also observed that all genes studied (TLR2, TLR4, CD14, MyD88) were up-regulated during DSS-induced inflammation, which was localized to the distal colon (Ortega-Cava et al. 2003). To understand how the normal intestinal epithelium dealt with continuous exposure to commensal Gram-positive bacteria, possessing PAMP including PGN and bacterial lipopeptides that are recognized by TLR2, Melmed et al. (2003) isolated human colonic epithelial cells by laser capture microscopy and used IEC lines (CaCo-2, T84, HT29) to analyse the expression of TLR2, TLR6, TLR1 and toll inhibitory protein (Tollip) mRNA by RT-PCR and quantitative real-time PCR. They found that colonic epithelial cells and LP lymphoid cells express low levels of TLR2 from both noninflamed and inflamed tissues, express high levels of the inhibitory 
molecule Tollip, and that IEC were unresponsive to TLR2 ligands. The observation that IEC express a low level of TLR2 was consistent with previous observations by Cario et al. (2002), as discussed above and by the Sanderson group, showing that TLR2 mRNA was highly expressed in PBMC and was present in all human IEC (Naik et al. 2001). These results indicate that TLR expressed on intestinal epithelium may be regulated by the bacterial flora and the host mucosal immune responses. TLR on IEC may play a role in the induction of an inflammatory response to bacterial pathogens and in the development of tolerance to non-pathogenic commensal organisms.

Intestinal bacteria, especially pathogenic ones, are believed to be taken up and actively translocated by specialized epithelial cells, M cells, which are derived from villous epithelial cells and located over PP. A study by Rescigno et al. (2001) found that DC, a crucial cell population in the recognition of bacterial antigens and in the defence against pathogens, express tight junction protein, and can open up the tight junction between epithelial cells and take up bacteria directly without compromising epithelial barrier function (Rescigno et al. 2001). As an initiator of adaptive immune response, mucosal DC either infiltrate the intestinal LP diffusely, or form sub-epithelial aggregates scattered along the small and large intestine (Hamada et al. 2002). DC are activated via TLR expressed on their surface. Liu et al. (2002) observed that different subsets of DC (myeloid $v$. lymphoid) express different TLR, with human myeloid preDC1, but not lymphoid pre-DC2 expressing TLR2 and TLR4, and lymphoid pre-DC2, but not myeloid pre-DC1 expressing high levels of TLR7 and TLR9. Activation of TLR induces expression of various cytokines by DC, such as TNF- $\beta$, IL-6, and IL-12. IL-12 induced by TLR can contribute to Th cell differentiation into Th1 effector cells (Liu et al. 2003). Recent evidence has indicated the differences in TLR expression on murine DC subsets. Using RT-PCR, the mRNA expression of most TLR, including TLR1, 2, 4, 6, 8 and 9, was detected on all DC. A difference in TLR3, 5 and 7 expression was found between $\mathrm{CD} 8 \alpha^{+}$and $\mathrm{CD} 8 \alpha^{-}$DC subsets (Edwards et al. 2003). Furthermore, the differential expression of TLR on DC was also found between different strains of mice (BALB/c v. C57BL/6) with a strong expression of TLR2, 4, 5 and 6 on DC from BALB/c mice and preferential expression of TLR9 in B6 mice (Liu et al. 2002). However, the functional significance of these differential expressions of TLR on DC and DC subsets remains to be further elucidated. It is apparent that TLR expressed on DC constitute a critical link between antigen recognition and the induction of $\mathrm{T}$ cell immunity. Triggering different TLR by various microbial stimuli may drive DC to assume distinct phenotypes and functions. Using LPS (TLR4 ligand) and PGN (TLR2 ligand), Re \& Strominger (2001) showed that human DC preferentially express IL-12 and IL-10 when stimulated through TLR4 and TLR2, respectively. Similar observations were made in an animal study, showing that LPS-stimulated DC produced high levels of IL-12, and low levels of IL-10, whereas DC exposed to PGN produced low levels of IL-12 and high levels of IL-10 (Qi et al. 2003). However, a recent study by Higgins et al. (2003) showed that signalling through the TLR4 in response to bacteria (Bordetella pertussis) activates IL-10 production from DC, suggesting that this TLR4 pathway may promote the development of IL-10-secreting Treg, suppressing the inflammatory response. It is clear that pattern recognition receptors on intestinal epithelinus and APC play an important role in mediating a continuing dialogue between host and gut microbiota and generating a proper response.

\section{Probiotics and gut defences}

As discussed above, gut flora is complex and colonization of the intestinal mucosa is required for normal development of the host's immune system. It is also possible that colonization with specific microflora in the gut may play a role in balancing the intestinal mucosal immune system, which may contribute to the induction and maintenance of immunological tolerance to other luminal antigens in the normal host or to the inhibition of the dis-regulated responses induced by luminal antigens in diseased hosts. Recent evidence demonstrates that commensal bacteria regulate the intestinal development and function (Hooper et al. 2002). Probiotics are live micro-organisms that are ingested to promote beneficial effects on health by altering indigenous microflora. The beneficial effects of probiotics can also be achieved through a variety of mechanisms including regulation of cytokine production, enhancement of $\operatorname{IgA}$ secretion, production of antibacterial substances and enhanced tight junction of the intestinal barrier to protect against intercellular bacterial invasion, and competition with pathogenic micro-organisms for enterocyte adherence. A recent study examined the basic molecular mechanisms of probiotics in regulating intestinal epithelial health and found that one probiotic, Lactobacillus rhamnosus GG, promoted the survival of intestinal epithelial cells through the activation of anti-apoptotic Akt/protein kinase B and inhibition of pro-apoptotic p38 MAP kinase pathways (Yan \& Polk, 2005). The result of this study provided a new insight into our understanding of the signal transduction pathways in enterocytes that are regulated by probiotics and intestinal commensal bacteria.

Lactobacilli derived from endogenous flora of normal donors have been used as probiotics in functional foods and as vaccine stimuli. Probiotic treatment has been used to prevent some infectious and allergic diseases and they also have the potential to be used in treating IBD. Growing evidence from both human studies and animal models of IBD indicate that an aberrant response to altered enteric flora plays a significant role in the development of the disease. There is also evidence that the alteration of bacterial colonization in mice may be associated with the development of inflammation in the intestine (in IL-10 knockout model; Madsen et al. 1999). Before the development of colonic injury, there was an increased level of adherence and translocation of aerobic bacteria and decreased adherence of Lactobacillus spp. in IL-10 knockout (IL-10 ${ }^{-1-}$ ) mice (Madsen et al. 1999). This decrease in adherence of Lactobacillus spp. occurred during the time when colonic histological injury developed. A repopulation of the intestinal lumen with normal levels of Lactobacillus spp. attenuated the development of IBD in these mice. These results suggest that restoring the balance between Lactobacillus spp. and other bacterial strains may prevent the development of IBD in IL-10 $0^{-1-}$ mice. In a recent double-blind, placebo-controlled trial using two probiotic strains (Lactobacillus-salivairus and Bifidobacterium-infantis) in the IL-10 ${ }^{-1-}$ mice model, McCarthy et al. (2003) reported that both probiotic strains significantly attenuated colitis in IL-10 $0^{-1-}$ mice. To examine the impact of probiotics on the host immune system, both mucosal and systemic cytokine profiles from different groups (placebo, Lactobacillusand Bifidobacterium-treated) were analysed. The results showed that PP lymphocytes produced significantly lower amounts 
of interferon- $\gamma$ (INF- $\gamma$ ), and TNF- $\alpha$ in the probiotic-treated group (B. infantis). There was also a significant reduction in the proinflammatory cytokine production by spleen cells in the probiotic groups. However, the production of the immunoregulatory cytokine TGF- $\beta$ remained unchanged, suggesting that the probiotic effect may be mediated by altering the balance between proinflammatory and regulatory cytokines (McCarthy et al. 2003; Table 1). It is possible that specific strains of healthy gut microbiota can induce the production of IL-10 and TGF- $\beta$, which possess an important regulatory role in the development of inflammatory and allergic responses and in the induction of oral tolerance.

To investigate whether bacteria modulate cytokine responses in Crohn's disease, Borruel et al. (2002) collected samples of intestinal mucosa from ten patients with Crohn's disease and five disease controls undergoing right hemi-colectomy, and cultured these mucosal samples with either non-pathogenic Escherichia coli, Lactobacillus casei, Lactobacillus bulgaricus, or Lactobacillus crispatusin. They found that the production of the pro-inflammatory cytokine TNF- $\alpha$ by inflamed Crohn's disease mucosa was significantly reduced by co-culture with $L$. casei or $L$. bulgaricus, suggesting that probiotics may interact with immunocompetent cells using the mucosal interface and locally modulate the production of proinflammatory cytokines.

\section{Prebiotics and host defence}

As stated, prebiotics which can occur naturally (breast milk) or be used as food additives (inulin-type fructans, galactooligosaccharides) are non-digestible oligosaccharides which enter the colon and are fermented to change the colonic environment (acid $\mathrm{pH}$ and increased SCFA) to stimulate the increased proliferation of certain commensal bacteria, bifidobacteria and lactobacillus, which function as probiotics to stimulate intestinal host defences (Moro et al. 2002). This indirect effect of prebiotics, e.g. an altered colonic milieu leading to stimulus of bifidobacteria and lactobacillus proliferation, has been considered as the primary role for prebiotics as a health-promoting dietary supplement. However, more recently several studies have suggested that prebiotics can also have a direct effect on the gut that does not require the proliferation of commensal (probiotic bacteria). The indirect and direct effects of prebiotics on the development of host defence will briefly be reviewed here.

Indirect effects of prebiotics. Prebiotic oligosaccharides are configured in such a way that the small intestinal enzymes cannot hydrolyse them for absorption. Accordingly, they enter the colon intact and provide the 'preferential food' for certain colonizing bacteria through the process of fermentation. Fermentation by colonizing bacteria produces metabolites that reduce the intraluminal $\mathrm{pH}$ to 5 and increase a variety of SCFA including lactic, butyric, propionic and acetic acid. These SCFA may have additional functions in the colon including acting as an energy source for colonocytes as well as a stimulus for some of the bacterial-epithelial 'crosstalk' cellular events, e.g. up-regulation of TLR expression (Zapolska-Downar et al. 2004). Several studies have demonstrated the specific effect of prebiotic oligosaccharides in achieving indirect response in the colon. In a study in the Journal of Nutrition (Bouhnik et al. 1997), various carbohydrates were incubated with colonic bacteria and the SCFA content was determined by HPLC analysis. Only those carbohydrates that fit the criteria of prebiotics (oligosaccharides) produced the fermentation pattern anticipated. In a similar study from Gastroenterology (Gibson et al. 1995), a variety of carbohydrates including starch, sucrose, oligosaccharides and inulin were used in fermentation studies with colonic bacteria. Again, the defined prebiotic carbohydrates (inulin and oligofructose) were the only sugars to produce an acid pH. Finally, in the Journal of Applied Microbiology (Sghir et al. 1998), actual colonic bacteria were determined in stool samples of individuals receiving prebiotics as a function of time. Over $21 \mathrm{~d}$, the percentage of total culturable counts of colonic bacteria increased strikingly to a high percentage of total colonic bacteria for lactobacillus and bifidobacteria. These studies strongly suggest that prebiotics by an indirect effect on the colon changed the luminal milieu to affect an increased proliferation of probiotic bacteria. However, despite this careful documentation of the indirect effect of prebiotics on colonic bacteria, additional clinical trials are needed to prove their effect on the host defence. For example, no long-term studies have been done to demonstrate the sustained effect of prebiotics or bacterial flora. It is inconceivable that over time other colonic bacteria can adjust to the fermentation products of prebiotics in order to use them as substrates for their own proliferation thereby negating the prebiotic effect. This phenomenon needs to be studied in the long-term clinical trials. In addition, it is assumed that, because prebiotics stimulate an increase in bifidobacteria and lactobacilli, the effect of this stimulation in treatment/prevention of disease is the same as that observed using probiotics therapeutically. Whereas this assumption is logical, it needs to be proven by multi-centre trials in patients just as those reported for probiotics.

Direct effects of prebiotics. In addition to the indirect effects of prebiotics on intestinal host defences, there are recent studies to suggest that prebiotics may act directly in the intestinal lumen to help protect the gut from infection, inflammation and diarrhoea. The best example of this direct effect is the inhibition of pathologic bacteria adherence by certain oligosaccharides (Dai et al. 2000). Pathologic bacteria colonize the colon by first adhering to glycoconjugates on glycoproteins and lipids on the microvillus membrane. This adherence is necessary before colonization and microbial-epithelial 'crosstalk' can occur. Prebiotics comprised of non-digestible oligosaccharides can enter the colon intact. Certain terminal sugars on these oligosaccharides, e.g. oligofructose, can then interfere with the receptors on bacteria by binding to the bacteria and preventing its attachment to the same sugar on microvillus glycoconjugates (Fig. 3). Without adherence, pathogens cannot act on the gut epithelium to cause disease.

Other direct effects of prebiotics on immune functions have recently been reported. Roller et al. (2004) have shown that prebiotics, independent of their probiotic effect, can directly stimulate an up-regulation of regulatory (IL-10) and protective (INF- $\gamma$ ) cytokines. These authors have also shown that prebiotics have a greater effect on the polymeric IgA response in the ileum and caecum than that of probiotics or synbiotics. These studies suggest that prebiotics by themselves may directly stimulate the mucosal immune system.

In addition, recent studies suggest that prebiotics can interfere with the inflammatory effects of pathogens in a necrotizing enterocolitis (NEC) animal model (Catala et al. 1999). Using germ-free neonatal quail, investigators can reproduce the inflammatory/necrotic gut changes seen in premature infants with NEC. This was done by monocontamining germ-free quails with bacteria isolated from human prematures with NEC. If, however, the quail was first exposed to prebiotics, the degree of intestinal 

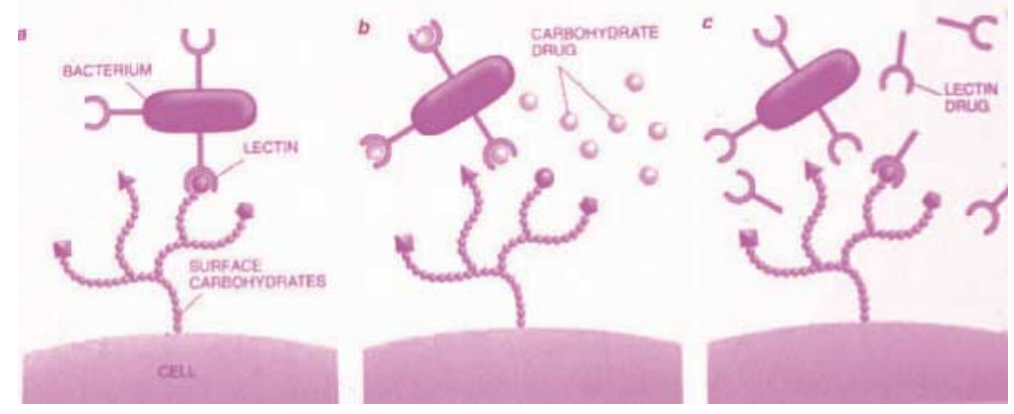

Fig. 3. This cartoon depicts a direct effect of prebiotics on host defence in the gut. Non-digested oligosaccharides enter the colon and free sugar moieties (galactose, fructose, etc.) competitively bind to receptors on pathogens, thus preventing their adherence to the same sugars in the microvillus surface glycoconjugates.

damage was reduced. These studies need to be repeated in other NEC models and in human clinical trials for validation.

\section{Summary}

In this review we have emphasized the importance of proper initial bacterial colonization of the gut in the development of intestinal host defences. We underscored the differences in gut lymphoid activity in germ-free $v$. colonized animals. As the field of bacterial-epithelial 'crosstalk' has emerged as an important research discipline for microbiologists, mucosal immunologists and gastroenterologists, we stressed that PAMP conserved on the colonizing bacteria interact with pattern recognition receptors on enterocytes and intestinal lymphoid cells to monitor an innate immune response followed by an adaptive immune response to invading pathogens. A family of pattern recognition receptors, TLR, has been shown to be important cellular/ molecular mediators of bacterial-epithelial 'crosstalk'. More recently, this same receptor family has been implicated in commensal activation of the developing mucosal immune system (oral tolerance, regulatory $\mathrm{T}$ cells stimulation, etc.) that prevent bacteria-induced gastrointestinal disease. Probiotics have been shown to stimulate the gut defences to prevents a variety of infectious and inflammatory diseases in children (e.g. rotavirus, gastroenteritis, etc.) and allergic diseases (e.g. atopic dermatitis). Finally, we have reviewed the effects of prebiotics stimulating intestinal host defences. Prebiotics work indirectly by altering the colonic milieu to favour stimulation of bifidobacteria and lactobacillus proliferation and presumably activation of host defences in a manner similar to probiotics. In addition, the direct effects of prebiotics have been shown to prevent pathologic colonization and to stimulate intestinal defences (pIgA release). However, additional multi-centre trials are needed to confirm the protective effects of prebiotics on the developing human gut defences.

\section{References}

Akira S, Takeda K \& Kaisho T (2001) Toll-like receptors: critical proteins linking innate and acquired immunity. Nat Immunol 2, 675-680.

Borruel N, Carol M, Casellas F, Antolin M, de Lara F, Espin E, Naval J, Guarner F \& Malagelada JR (2002) Increased mucosal tumour necrosis factor $\alpha$ production in Crohn's disease can be downregulated ex vivo by probiotic bacteria. Gut 51, 659-664.

Bouhnik Y, Flourie B, D'Agay-Abensour L, Pochart P, Gramet G, Durand M \& Rambaud JC (1997) Administration of transgalacto-oligosaccharides increases fecal bifidobacteria and modifies colonic fermentation metabolism in healthy humans. $J$ Nutr 127, 444-448.

Caramalho I, Lopes-Carvalho T, Ostler D, Zelenay S, Haury M \& Demengeot J (2003) Regulatory T cells selectively express toll-like receptors and are activated by lipopolysaccharide. J Exp Med 197, 403-411.

Cario E \& Podolsky DK (2000) Differential alteration in intestinal epithelial cell expression of toll-like receptor 3 (TLR3) and TLR4 in inflammatory bowel disease. Infect Immun 68, 7010-7017.

Cario E, Brown D, McKee M, Lynch-Devaney K, Gerken G \& Podolsky DK (2002) Commensal-associated molecular patterns induce selective toll-like receptor-trafficking from apical membrane to cytoplasmic compartments in polarized intestinal epithelium. Am J Pathol 160, 165-173.

Catala I, Butel MJ, Bensaada M, Popot F, Tessedre AC, Rimbault A \& Szylit O (1999) Oligofructose contributes to the protective role of bifidobacteria in experimental necrotizing enterocolitis in quails. $\mathrm{J} \mathrm{Med}$ Microbiol 48, 89-94.

Cebra JJ (1999) Influences of microbiota on intestinal immune system development. Am J Clin Nutr 69, 1046s-1051s.

Clavano NR (1982) Mode of feeding and its effect on infant mortality and morbidity. J Trop Pediatr 28, 287-293.

Dabbagh K \& Lewis DB (2003) Toll-like receptors and T-helper-1/Thelper-2 responses. Curr Opin Infect Dis 16, 199-204.

Dai D, Nanthkumar N, Newberg D \& Walker AW (2000) Role of oligosaccharides and glycoconjugates in intestinal host defence. J Pediatr Gastroenterol Nutr 30, S23-S33.

Edwards AD, Diebold SS, Slack EM, Tomizawa H, Hemmi H, Kaisho T, Akira S \& Reise Sousa C (2003) Toll-like receptor expression in murine DC subsets: lack of TLR7 expression by CD8 alpha+ DC correlates with unresponsiveness to imidazoquinolines. Eur J Immunol 33, $827-833$.

Falk PG, Hooper LV, Midtvedt T \& Gordon JI (1999) Creating and maintaining the gastrointestinal ecosystem: what we know and need to know from gnotobiology. Microbiol Mol Biol Rev 62, 1157-1170.

Fusunyan RF, Nanthakumar NN, Baldeon ME \& Walker WA (2001) Evidence for an innate immune response in the immature human intestine: toll-like receptors on fetal enterocytes. Pediatr Res 49, 589-593.

Gewirtz AT, Navas TA, Lyons S, Godowski PJ \& Madara JL (2001) Cutting edge: bacterial flagellin activates basolaterally expressed tlr5 to induce epithelial proinflammatory gene expression. J Immunol 167, $1882-1885$.

Gibson GR, Beatty ER, Wang X \& Cummings JH (1995) Selective stimulation of bifiodobacteria in the human colon by oligofructose and inulin. Gastroenterology 108, 975-982.

Hamada H, Hiroi T, Nishiyama Y, et al. (2002) Identification of multiple isolated lymphoid follicles on the antimesenteric wall of the mouse small intestine. J Immunol 168, 57-64. 
Hershberg RM \& Mayer LF (2000) Antigen processing and presentation by intestinal epithelial cells - polarity and complexity. Immunol Today 21, 23-28.

Higgins SC, Lavelle EC, McCann C, Keogh B, McNeela E, Byrne P, O'Gorman B, Jarnicki A, McGuirk P \& Mills KH (2003) Toll-like receptor 4-mediated innate IL-10 activates antigen-specific regulatory $\mathrm{T}$ cells and confers resistance to Bordetella pertussis by inhibiting inflammatory pathology. J Immunol 171, 3119-3122.

Hooper LV, Wong MH, Thelin A, Hansson L, Falk PG \& Gordon JI (2002) Molecular analysis of comensal host-microbial relationships in the intestine. Science 291, 881-884.

Julia V, McSorley SS, Malherbe L, Breittmayer JP, Girard-Pipau F, Beck A \& Glaichenhaus N (2000) Priming by microbial antigens from the intestinal flora determines the ability of CD4+ $\mathrm{T}$ cells to rapidly secrete IL-4 in BALB/c mice infected with Leishmania major. J Immunol 165, 5637-5645.

Khoo UY, Proctor IE \& Macpherson AJ (1997) CD4+ T cell down-regulation in human intestinal mucosa: evidence for intestinal tolerance to luminal bacterial antigens. J Immunol 158, 3626-3634.

Lefrancois L \& Goodman T (1989) In vivo modulation of cytolytic activity and Thy-1 expression in TCR-gamma delta+ intraepithelial lymphocytes. Science 243, 716-718.

Liu T, Matsuguchi T, Tsuboi N, Yajima T \& Yoshikai Y (2002) Differences in expression of toll-like receptors and their reactivities in dendritic cells in BALB/c and C57BL/6 mice. Infect Immun 70, 6638-6645.

Liu YJ, Kanzler H, Soumelis V \& Gilliet M (2003) Dendritic cell lineage, plasticity and cross-regulation. Nat Immunol 2, 585-589.

McCarthy J, O'Mahony L \& O'Callaghan L (2003) Double blind, placebo controlled trial of two probiotic strains in interleukin 10 knockout mice and mechanistic link with cytokine balance. Gut 52, 975-980.

Macpherson AJ, Gatto D, Sainsbury E, Harriman GR, Hengartner H \& Zinkernagel RM (2001) A primitive $\mathrm{T}$ cell-independent mechanism of intestinal mucosal $\operatorname{IgA}$ responses to commensal bacteria. Science 288, 2222-2226.

Madsen KL, Doyle JS, Jewell LD, Tavernini MM \& Fedorak RN (1999) Lactobacillus species prevents colitis in interleukin 10 gene-deficient mice. Gastroenterology 116, 1107-1114.

Medzhitov R, Preston-Hurlburt P \& Janeway CA Jr (1997) A human homologue of the Drosophila toll protein signals activation of adaptive immunity. Nature 388, 394-397.

Melmed G, Thomas LS, Lee N, Tesfay SY, Lukasek K, Michelsen KS, Zhou Y, Hu B, Arditi M \& Abreu MT (2003) Human intestinal epithelial cells are broadly unresponsive to toll-like receptor 2-dependent bacterial ligands: implications for host-microbial interactions in the gut. J Immunol 170, 1406-1415.

Moreau MC \& Corthier G (1988) Effect of the gastrointestinal microflora on induction and maintenance of oral tolerance to ovalbumin in $\mathrm{C} 3 \mathrm{H}$ / HeJ mice. Infect Immun 56, 2766-2768.

Moreau MC \& Gaboriau-Routhiau V (1996) The absence of gut flora, the doses of antigen ingested and aging affect the long-term peripheral tolerance induced by ovalbumin feeding in mice. Res Immunol 147, 49-59.

Moreau MC, Ducluzeau R, Guy-Grand D \& Muller MC (1988) Increase in the population of duodenal immunoglobulin A plasmocytes in mice associated with different living or dead bacterial strains of intestinal origin. Infect Immun 21, 532-539.

Moro G, Minoli I, Mosca M, Fanaro S, Jelinek J, Stahl B \& Boehm G (2002) Dosage-related bifidogenic effects of galacto- and fructooligosaccharides in formula-fed term infants. J Pediatr Gastroenterol Nutr 34, 291-295.

Mottet C, Uhlig HH \& Powrie F (2003) Cutting edge: cure of colitis by CD4+CD25+ regulatory T cells. J Immunol 170, 3939-3943.

Mushegian A \& Medzhitov R (2001) Evolutionary perspective on innate immune recognition. J Cell Biol 155, 705-710.

Naik S, Kelly EJ, Meijer L, Pettersson S \& Sanderson IR (2001) Absence of toll-like receptor 4 explains endotoxin hyporesponsiveness in human intestinal epithelium. J Pediatr Gastroenterol Nutr 32, 449-453.
Nanthakumar NN, Dai D, Newburg DS \& Walker WA (2003) The role of indigenous microflora in the development of murine intestinal fucosyland sialyltransferases. FASEB $J$ 17, 44-46.

Ortega-Cava CF, Ishihara S, Rumi MA, Kawashima K, Ishimura N, Kazumori H, Udagawa J, Kadowaki Y \& Kinoshita Y (2003) Strategic compartmentalization of toll-like receptor 4 in the mouse gut. J Immunol 170, 3977-3985.

Qi H, Denning TL \& Soong L (2003) Differential induction of interleukin10 and interleukin-12 in dendritic cells by microbial toll-like receptor activators and skewing of T-cell cytokine profiles. Infect Immun 71, 3337-3342.

Re F \& Strominger JL (2001) Toll-like receptor 2 (TLR2) and TLR4 differentially activate human dendritic cells. $J$ Biol Chem 276, 37692-37699.

Read S, Malmstrom V \& Powrie F (2000) Cytotoxic T lymphocyte-associated antigen 4 plays an essential role in the function of CD25+CD4+ regulatory cells that control intestinal inflammation. J Exp Med 192, 295-302.

Rescigno M, Urbano M, Valzasina B, Francolini M, Rotta G, Bonasio R, Granucci F, Kraehenbuhl JP \& Ricciardi-Castagnoli P (2001) Dendritic cells express tight junction proteins and penetrate gut epithelial monolayers to sample bacteria. Nat Immunol 2, 361-367.

Roller M, Rechkemmer \& Watzl B (2004) Prebiotic inulin enriched with oligofructose in combination with the probiotics Lactobacillus rhamnosus and Bifidobacterium lactis modulates intestinal immune functions in rats. $J$ Nutr 134, 153-156.

Savidge TC, Smith MW, James PS \& Aldred P (1991) Salmonellainduced M-cell formation in germ-free mouse Peyer's patch tissue. Am J Pathol 139, 177-184.

Sghir A, Chow JM \& Mackie RI (1998) Continuous culture selection of bifidobacteria and lactobacilli from human faecal samples using fructooligosaccharide as selective substrate. J Appl Microbiol 85, $769-777$.

Shevach EM (2000) Suppressor T cells: rebirth, function and homeostasis. Curr Biol 10, R572-R578.

Steege JC, Buurman WA \& Forget PP (1997) The neonatal development of intraepithelial and lamina propria lymphocytes in the murine small intestine. Dev Immunol 5, 1121-1128.

Sudo N, Sawamura S, Tanaka K, Aiba Y, Kubo C \& Koga Y (1997) The requirement of intestinal bacterial flora for the development of an $\mathrm{IgE}$ production system fully susceptible to oral tolerance induction. J Immunol 159, 1739-1745.

Suri-Payer E, Amar AZ, Thornton AM \& Shevach EM (1998) $\mathrm{CD} 4+\mathrm{CD} 25+\mathrm{T}$ cells inhibit both the induction and effector function of autoreactive $\mathrm{T}$ cells and represent a unique lineage of immunoregulatory cells. J Immunol 160, 1212-1218.

Talham GL, Jiang HQ, Bos NA \& Cebra JJ (1999) Segmented filamentous bacteria are potent stimuli of a physiologically normal state of the murine gut mucosal immune system. Infect Immun 67, 1992-2000.

Wannemuehler MJ, Kiyono H, Babb JL, Michalek SM \& McGhee JR (1992) Lipopolysaccharide (LPS) regulation of the immune response: LPS converts germfree mice to sensitivity to oral tolerance induction. J Immunol 129, 959-965.

Yamanaka T, Helgeland L, Farstad IN, Fukushima H, Midtvedt T \& Brandtzaeg P (2005) Microbial colonization drives lymphocyte accumulation and differentiation in the follicle-associated epithelium of Peyer's patches. J Immunol 170, 816-822.

Yan F \& Polk DB (2002) Probiotic bacterium prevents cytokine-induced apoptosis in intestinal epithelial cells. J Biol Chem 277, 50959-50965.

Yoshioka H, Iseki K \& Fujita K (1983) Development and differences of intestinal flora in the neonatal period in breast-fed and bottle-fed infants. Pediatrics 72, 317-321.

Zapolska-Downar D, Siennicka A, Kaczmarczyk M, Kolodieg B \& Nruszewicz M (2004) Butyrate inhibits cytokine-induced VCAM-1 and ICAM-1 expression in cultured endothelial cells: the role of NF$\kappa \mathrm{B}$ and PPAR $\alpha$. J Nutr Biochem 15, 220-228. 